\title{
Assessment tools to evaluate Activities of Daily Living (ADL) and Instrumental Activities of Daily Living (IADL) in older adults: A systematic review
}

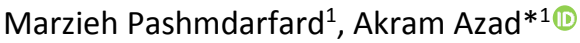 \\ Received: 12 Aug 2019 \\ Published: 13 Apr 2020
}

Abstract

Background: Given the increasing population of older adults in different societies, it is important to take into account the needs of them. In this regard, the most important things that are closely related to their quality of life are their ability in evaluating Activity of Daily Living (ADL) and Instrumental Activity of Daily Living (IADL) performances. The aims of the present study were to identify the outcome measures specific to the ADL and IADL for older adults and to investigate the psychometric properties of these measures.

Methods: This is a systematic review done on the articles published between June 2019 and February 2019. Articles in English language from these database included: Medline, PubMed, Google Scholar, CINAHL, OVID Medline, Cochrane, ProQuest, Up to Date, Web of Science, OT search, OT direct, Pedro, SID, Magiran, Iran Medex, MEDLIB and Iran doc. English keywords included: "Activity of Daily Living (ADL)", "Instrumental Activity of Daily Living (IADL)", "assessment", "evaluation”, "aging", "ageing", "older adults", "elders", "Basic Activity of Daily Living (BADL)", "Advanced Activity of Daily Living (AADL)", "basic functions", "self-care", "mobility", "independency", "dependency", "occupational therapy", "physical therapy", "rehabilitation". The Consensusbased Standards for the selection of health Measurement Instruments (COSMIN) checklist were employed to investigate the psychometric properties of the studies.

Results: Of the initial 482 studies considered, 13 studies met the inclusion criteria that assess the ADL and IADL performance of older adults. In this regard, 8 outcome measures were found especially for ADL assessment and 5 for IADL assessment.

Conclusion: Most of the assessment tools are performance-based and have been developed in especial contexts and especial groups of older adults. Some have been used frequently in different contexts but some were used less than others. None of these measures has been developed in Iran. So, for better assessment and having better intervention plans for older adults in Iran, it is suggested to develop an instrument that is especially designed for Iranian context.

Keywords: Elderly, Evaluative, Everyday life, Review

Conflicts of Interest: None declared

Funding: None

*This work has been published under CC BY-NC-SA 1.0 license.

Copyright $($ Iran University of Medical Sciences

Cite this article as: Pashmdarfard M, Azad A. Assessment tools to evaluate Activities of Daily Living (ADL) and Instrumental Activities of Daily Living (IADL) in older adults: A systematic review. Med J Islam Repub Iran. 2020 (13 Apr); 34:33. https://doi.org/10.47176/mjiri.34.33

Introduction

Aging refers to the process of becoming older, which occurs during a series of changes over time such as physi-
Corresponding author: Dr Akram Azad, azad.a@iums.ac.ir

1. Rehabilitation Research Center, Department of Occupational Therapy, School of Rehabilitation Sciences, Iran University of Medical Sciences, Tehran, Iran

\section{$\uparrow$ What is "already known" in this topic:}

Assessment tools for evaluating Activities of Daily Living (ADL) and Instrumental Activities of Daily Living (IADL) are very important. These instruments are developed in especial context and especial community. Given the increase in the number of the ageing population, it is important to identify the barriers and performance problems of this community by these tools.

$\rightarrow$ What this article adds:

This artic introduces 8 assessment tools for assessing ADL and 5 assessment tools for IADL of elderly people. These tools were not specific to Iranian culture and were not designed in Iran. Therefore, it is better to have a step towards developing a suitable tool for assessing ADL and IADLs in Iranian elderly. 
cal, mental and social changes (1). Aging is one of the greatest known causes for many human diseases (2). According to the United Nation's (UN) population estimates, the world's population over 65 will reach 2 billion in 2050 $(28 \%)$ in the world. Concerning the population growth within recent decades, the country's future will be an aging population (1). $30 \%$ of people aged $75-79$ and $40 \%$ of people aged 85 and over have at least one disability from a specific disease, such as stroke, Parkinson's disease $(1,3)$. Therefore, health and rehabilitation systems in the country should take into account the projections and plans necessary to improve the quality of life of this group, especially rehabilitation services to improve the performance of everyday life activities. Successful performance in activities of daily living to have an independent life is essential for every age (4). According to the definitions of medical and rehabilitation texts, the Activities of Daily Living (ADL) and Instrumental Activities of Daily Living (IADL) are described as follows:

The ADL refers to activities oriented toward taking care of one's own body. These activities are fundamental to living in a social world; they enable basic survival and wellbeing, such as bathing, toileting, dressing and eating (5).

The IADL refers to activities to support daily life within the home and community that often require more complex interactions than those used in ADLs. Examples of such activities include financial management, housekeeping, shopping for groceries, making telephone calls, and taking medication (5). The ADL and IADL functions are important to older adults, and IADL autonomy plays an important role in "successful" aging (6). Older adults experience ADL and IADL disabilities through two pathways: 1) a catastrophic event, such as a hip fracture, or 2) progressive decline in the brain functions (7). Doing day-today functions, especially IADLs, has a significant correlation with executive functions such as planning, working memory, attention, problem solving, verbal reasoning and mental flexibility $(8,9)$. Successful performance in ADL and IADLs are significant health indicators that can predict mild cognitive impairments, dementia, and mortality in older adults $(10,11)$.

To have a comprehensive planning for the older adults to be independent in ADL and IADLs, the rehabilitation specialists, aging medicine and nursing rehabilitation specialists should have an accurate understanding of all types of effective measures of ADL and IADL in older adults. Therefore, the purpose of this study was to review the assessment tools of ADL and IADL functions in older adults to have a common language between rehabilitation specialists, aging medicine and nursing rehabilitation specialists.

\section{Methods}

\section{Search Strategy}

The present study is a systematic review that aims to explore a variety of assessment tools of ADL and IADL in older adults regardless of time limitations. For data gathering, two researchers (a Librarian and an Occupational Therapist who is working in the field of aging) searched the articles based on keywords and English and Persian database sources individually. English and Persian Electronic databases include Medlin, PubMed, Google scholar, CINAHL, OVID Medline, Cochrane, ProQuest, Up to Date, Web of Science, OT search, OT direct, Pedro, SID, MagIran, Iran Medex, MEDLIB and Iran doc.

\section{Search Terms}

The English keywords used individually or in combination (according to the MeSH) were as follows: "Activity of Daily Living (ADL)", "Instrumental Activity of Daily Living (IADL)", "assessment", “evaluation", “aging”, "ageing", "older adults", "elders", "Basic Activity of Daily Living (BADL)", "Advanced Activity of Daily Living (AADL)", "basic functions", "self-care", "mobility", "independency", "dependency", "occupational therapy", "physical therapy", "rehabilitation". The search strategy is

Table 1. Search strategy for different database

\begin{tabular}{|c|c|c|}
\hline & Database & Search strategy \\
\hline 1 & Medline & $\begin{array}{l}\text { (exp ACTIVITIES OF DAILY LIVING (ADL)) AND(exp AGING OR exp ASSESSMENT) } \\
\text { (exp INSTRUMENTAL ACTIVITIES OF DAILY LIVING (IADL) ) AND (exp AGING, OR ASSESSMENT)1 } \\
\text { (exp CHILDREN) AND (exp SOCIAL PARTICIPATION) } \\
\text { (1 AND 2) OR (1 AND 3) - limited to human, and English and no limitation in year. (inclusive) }\end{array}$ \\
\hline 2 & Psychinfo & $\begin{array}{l}\text { (exp Activities of Daily Living (ADL)) AND (exp Aging OR exp Ageing OR exp Assessment OR exp older adults OR exp elders OR } \\
\text { exp ADL) } \\
\text { (exp aging OR exp Instrumental Activities of Daily Living (IADL) ) AND (exp aging OR exp older adilts) } \\
\text { (exp aging OR exp ADL) AND (exp "IADL (activity)") } \\
\text { (1 AND 2) OR (1 AND 3) - limited to human, and English and no limitation in year. (inclusive) }\end{array}$ \\
\hline 3 & Cinahl & $\begin{array}{l}\text { (exp AGING) AND(exp ACTIVITIES OF DAILY LIVING(ADL) OR exp INSTRUMENTAL ACTIVITIES OF DAILY } \\
\text { LIVING(IADL) OR exp ASSESSMENT) } \\
\text { (exp AGING) AND (exp ADL, IADL OR exp ACTIVITIES OR exp ASSESSMENT TOOLS) } \\
\text { (exp ADVANCED ADL) AND (exp OLDER ADULTS) } \\
\text { (1 AND 2) OR (1 AND 3) - limited to research and no limited in years (Inclusive) }\end{array}$ \\
\hline 4 & Embase & $\begin{array}{l}\text { ('AGING/exp OR AGEING OR (AGING AND (AGEING/exp OR ELDERS))) AND (ADL/exp OR IADL) AND (ASSESSMENT) } \\
\text { ('AGING/exp OR AGIENG) AND ('occupational therapy'/exp OR 'occupational therapy' OR (occupational AND ('therapy'/exp OR } \\
\text { therapy))) AND (ASSESSMENT) } \\
\text { ('AGONG /exp OR 'ELDERS'OR (older AND (ADULT/exp OR elder))) AND ('ADL'/exp OR IADL) AND (ASSESSMENT) } \\
\text { ('daily life activity'/exp OR 'instrumental daily life activity') AND ('Aging /exp OR 'ageing' OR (older AND (elder/exp OR older } \\
\text { adults))) AND (assessment) } \\
\text { (1 AND 2) OR (1 AND 3) - limited to research and no limited in years. }\end{array}$ \\
\hline 5 & $\begin{array}{l}\text { Google scholar and } \\
\text { other resource }\end{array}$ & $\begin{array}{l}\text { "Activities of Daily Living (ADL)" AND ("Instrumental Activities of Daily Living (IADL) " OR "aging" OR " older adults" OR } \\
\text { "elders") AND ("Assessment" or scale or evaluation)- No limitation in years. }\end{array}$ \\
\hline
\end{tabular}


seen in Table 1.

\section{Study selection and data extraction}

All selected articles were assessed by two independent reviewers based on inclusion and exclusion criteria. The inclusion criteria included the articles on assessment tools of ADL and IADL in older adults, and having access to the abstract or full text of the articles. The exclusion criteria were the articles in other than Persian and English language, and the articles on younger populations. Disagreements between reviewers about the articles were resolved by consensus. If consensus was not reached the final decision was made by a third independent reviewer.

\section{Results}

Based on the definitions given in medical and rehabilitation texts, ADL and IADL were differentiated from each other $(5,7)$. The assessment tools for ADL and IADL of older adults categorized into two groups of ADL assessment tools and IADL assessment tools. Figure 1 shows the PRISMA flow diagram for the studies included in the present review (12). Of the initial 482 studies considered, 13 studies met the inclusion criteria, which assess the ADL and IADL performance of older adults. In this regard, 8 outcome measures were especially for ADL assessment and 5 were especially for IADL assessment. The characteristics of the studies were presented in Table 2.

\section{Results of the measurement properties}

The risk of bias COSMIN checklist was used to investigate the psychometric properties of the measures. This standard can be used either to assess the methodological quality of a study or to compare the properties of various measurement instruments in a systematic review, which was done by two independent reviewers (13). In this study, the measurement properties are divided into two domains: reliability and validity. Concerning the psychometric properties proposed by Terwee et al (14) each issue was rated as positive ' + ' (adequate description or value or measure or argument related to the psychometric property), negative '-' (inadequate or values below the accepted standards for the psychometric property), indeterminate '?' (Doubtful methods or measures or design) or absent ' 0 ' (no information available about the psychometric property). The results of psychometric properties of measures are presented in Table 3 .

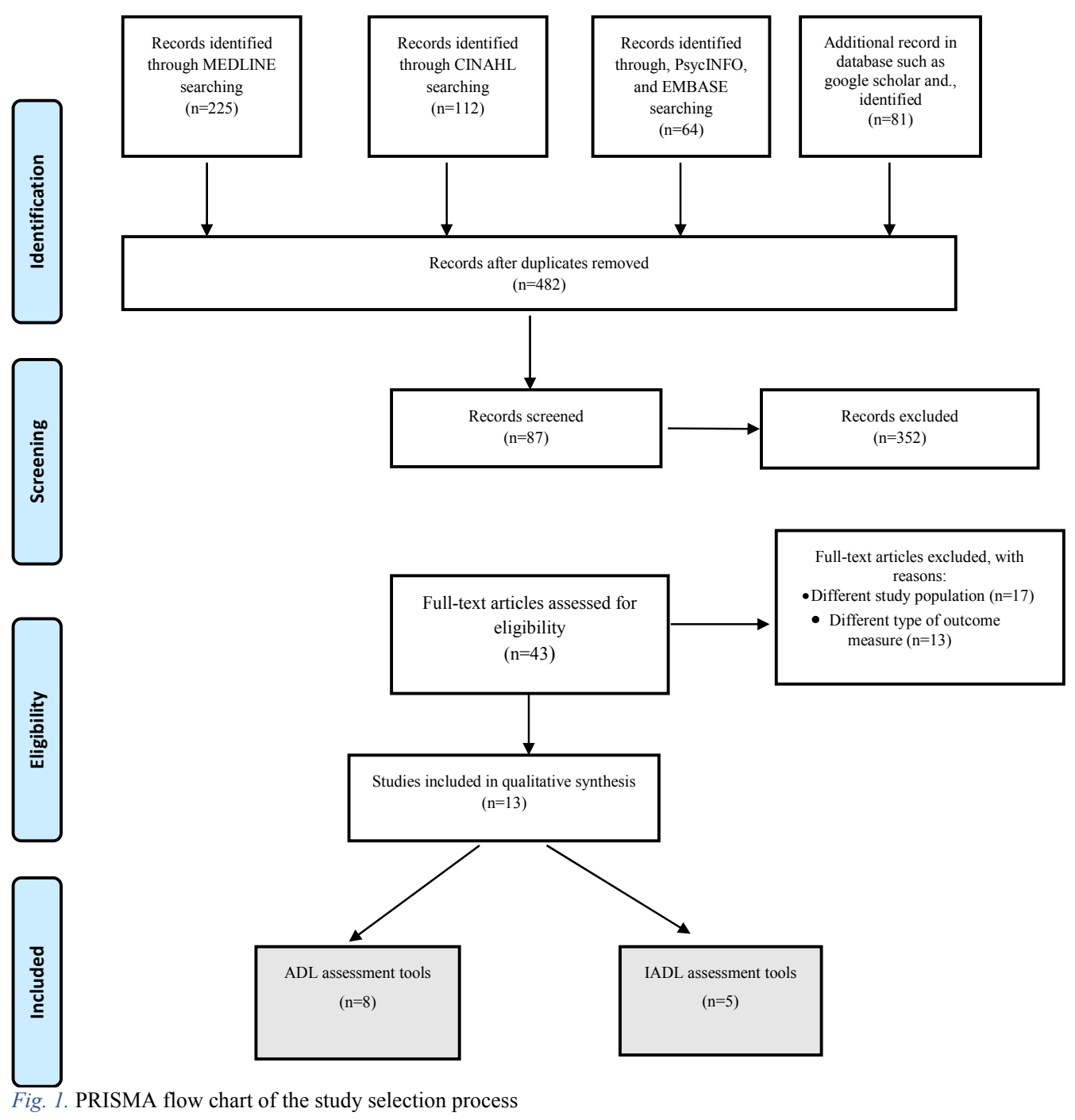


Table 2. Characteristics of included measures

\begin{tabular}{lcc}
\hline $\begin{array}{l}\text { PROM* } \\
\text { (reference to first article) }\end{array}$ & $\begin{array}{c}\text { Target } \\
\text { population }\end{array}$ & $\begin{array}{c}\text { Mode of administration } \\
\text { (e.g. self-report, inter- } \\
\text { view-based, parent/proxy } \\
\text { Report etc.) }\end{array}$ \\
\hline Barthel Index (BI) & $\begin{array}{c}\text { Stroke, } \\
\text { other neuromuscular, } \\
\text { musculoskeletal disor- } \\
\text { ders, } \\
\text { oncology patients }\end{array}$ & $\begin{array}{c}\text { Self-report, direct observation. } \\
\text { ononey \& Barthel (1995) }\end{array}$ \\
Katz Index of Independence in & $\begin{array}{c}\text { Older adults in the } \\
\text { community and all care } \\
\text { scttings }\end{array}$ & Self-report, direct observation. \\
Katz et al (1959) & &
\end{tabular}

Katz et al (1959) ty and all care settings

Stroke, TBI, SCI, MS, elderly individuals undergoing inpatient rehabilitation, children as young as 7 years old
Performance based (direct observation of the evaluated function)

(FIM) Instrument

American Academy of Physical Medicine and Rehabilitation \& American Congress of Rehabilitation Medicine

\section{Keith et al (1987)}

\author{
Activities of Daily Living (ADL)
}

Profile

TBI, Stroke

\section{Dutil et al (1990)}

\section{Activities of Daily Living Questionnaire (ADLQ)}

Johnson et al (2004) (direct ed function), and nterviews with the person or other people (individual's caregivers)

Informant-based tividual with cogn especially Alzheimer

Recall

period

Self-report: 2-5 minutes ;

direct observation: 20

minutes,

also according to pa-

tient's abilities and toler-

ance

Self-report: 2-5 minutes; direct observation: 20 minutes,

also according to pa-

tient's abilities and toler-

ance

It take between 30-45 minutes to administer and score, with 7 minutes to gather demographic information.

\section{Basic ADL function}

-

Total score: between 6 (maximum performance) and 0 (lack of performance).

Also: score of 6 (full function),

4 (moderate impairment), and

2 or less (severe functional impairment)

18 items that evaluate 6 Each item is scored on a 7functional areas, The 13 point Likert scale, and the items are named as score indicates the amount of Motor-FIM and its 5 assistance required to perform items are named as each item (1=total assistance in Cognitive-FIM

each item $(1=$ total assistance in
all areas, $7=$ total independence in all areas).

A final summed score is created and ranges from 18-126 (18 represents complete dependence/total assistance, 126 represents complete independence).

Performance-based evaluation 30-60 minutes. (direct observation of the evaluat- When administered in preparation for dischar from a rehabilitation from a rehabilitation
hospital up to 7 hours may be required.

5-10 minutes
Assesses the ADL func- 0 (full independency) to

tion of in three personal 3 (full dependency)

environments (with 6

items), Home (with 5

items), and the Commu-

nity (with 9 items)

6 areas
0 (no problem) to

3 (need help for completion and long-term)
Original Available

language translations

\begin{tabular}{|c|c|}
\hline English & $\begin{array}{l}\text { Portuguese, Brit- } \\
\text { ish, Dutch, Ger- } \\
\text { man, Taiwanese, } \\
\text { Turkish, Chinese } \\
\text { (Hong Kong), } \\
\text { Persian. }\end{array}$ \\
\hline $\begin{array}{l}\text { American } \\
\text { (English) }\end{array}$ & $\begin{array}{l}\text { Brazilian, Turk- } \\
\text { ish, Swedish, } \\
\text { Persian }\end{array}$ \\
\hline English & $\begin{array}{l}\text { German, Italian, } \\
\text { Spanish, Swedish, } \\
\text { Finnish, Portu- } \\
\text { guese, African, } \\
\text { Turkish, French, } \\
\text { Persian }\end{array}$ \\
\hline Canadian & $\begin{array}{l}\text { Canadian, } \\
\text { French }\end{array}$ \\
\hline English & $\begin{array}{l}\text { American, Chi- } \\
\text { nese, Spanish, } \\
\text { Brazilian, Chilean }\end{array}$ \\
\hline
\end{tabular}




\begin{tabular}{|c|c|c|c|c|c|c|c|}
\hline $\begin{array}{l}\text { PROM* } \\
\text { (reference to first article) }\end{array}$ & $\begin{array}{c}\text { Target } \\
\text { population }\end{array}$ & $\begin{array}{l}\text { Mode of administration } \\
\text { (e.g. self-report, interview-based, } \\
\text { parent/proxy Report etc.) }\end{array}$ & $\begin{array}{l}\text { Recall } \\
\text { period }\end{array}$ & $\begin{array}{l}\text { Sub/scales, } \\
\text { Number of items }\end{array}$ & $\begin{array}{c}\text { Range of } \\
\text { scores/scoring }\end{array}$ & $\begin{array}{l}\text { Original } \\
\text { language }\end{array}$ & $\begin{array}{l}\text { Available } \\
\text { translations }\end{array}$ \\
\hline $\begin{array}{l}\text { Australian Therapy Outcome } \\
\text { Measures (AusTOMs) } \\
\text { Perry et al (2004) }\end{array}$ & $\begin{array}{l}\text { Client profiles and } \\
\text { patterns of services } \\
\text { provision across health } \\
\text { care settings }\end{array}$ & Performance-based evaluation tool & Not mentioned & $\begin{array}{l}\text { There are } 6 \text { speech pa- } \\
\text { thology scales, } 9 \text { physio- } \\
\text { therapy scales and } 12 \\
\text { occupational therapy } \\
\text { scales. }\end{array}$ & $\begin{array}{l}0 \text { (sever impairment, complete } \\
\text { difficulty) to } \\
5 \text { (no impairment, no difficulty) }\end{array}$ & Australian & $\begin{array}{l}\text { English, } \\
\text { Swedish }\end{array}$ \\
\hline $\begin{array}{l}\text { Melbourne Low-Vision ADL Index } \\
\text { (MLVAI) } \\
\text { Haymes et al (2001) }\end{array}$ & $\begin{array}{l}\text { Individuals with visual } \\
\text { impairment }\end{array}$ & $\begin{array}{l}\text { Performance-based evaluation tool } \\
\text { (desk based clinical assessment) }\end{array}$ & Not mentioned & $\begin{array}{l}\text { Consisting } 18 \text { observa- } \\
\text { tional items in the field } \\
\text { of ADL functions, and } 9 \\
\text { items for self-care activi- } \\
\text { ties }\end{array}$ & $\begin{array}{l}\text { Scoring for each item is based on } \\
\text { independency, speed and accuracy } \\
\text { of performance on a five descrip- } \\
\text { tive Likert scale }(0-4)\end{array}$ & English & English \\
\hline $\begin{array}{l}\text { Self-Assessment Parkinson's disease } \\
\text { Disability Scale (SPDDS) }\end{array}$ & Parkinson & Self-report (paper-pencil) & 5 minutes & $\begin{array}{l}24 \text { items that assess the } \\
\text { ADL performance }\end{array}$ & $\begin{array}{l}5 \text { (ability to do work alone and } \\
\text { without difficulty) to } \\
1 \text { (unable to do activity) }\end{array}$ & English & $\begin{array}{l}\text { English, } \\
\text { Serbian }\end{array}$ \\
\hline Brown (1989) & & & & & & & \\
\hline $\begin{array}{l}\text { Frenchay Activities Index (FAI) } \\
\text { Holbrook \& Skilbeck (1983) }\end{array}$ & Stroke & Interview & 5 minutes & $\begin{array}{l}15 \text { items that cover three } \\
\text { areas of domestic chores } \\
\text { Work/Leisure, and Out- } \\
\text { door activities. }\end{array}$ & $\begin{array}{l}1 \text { (lowest level of activity) to } \\
4 \text { (highest level of activity) }\end{array}$ & English & $\begin{array}{l}\text { English, } \\
\text { Chinese, } \\
\text { Dutch }\end{array}$ \\
\hline $\begin{array}{l}\text { Activities of Daily Living (IADL) } \\
\text { Profile Instrumental } \\
\text { Bottar et al (2010) }\end{array}$ & TBI & $\begin{array}{l}\text { Performance-based evaluation (direct } \\
\text { observation of the evaluated func- } \\
\text { tion), and semi-structured interviews } \\
\text { with the person or other people (indi- } \\
\text { vidual's caregivers }\end{array}$ & $30-60$ minutes & 29 items in 8 areas & $\begin{array}{l}0 \text { (full independency) to } \\
3 \text { (full dependency) }\end{array}$ & English & Canadian \\
\hline $\begin{array}{l}\text { Lawton Instrumental Activities of } \\
\text { Daily Living Scale (LIADL) } \\
\text { Lawton and Brody (1969) }\end{array}$ & $\begin{array}{l}\text { Older adults but isn't } \\
\text { useful for institutional- } \\
\text { ized older adults }\end{array}$ & Self-report & 10-15 minutes & $\begin{array}{l}8 \text { activities related to } \\
\text { IADL }\end{array}$ & $\begin{array}{l}\text { Scoring scale is zero and one. } \\
\text { Sum of the scores is varied from } 0 \\
\text { (low function, dependence) to } \\
8 \text { (high function, independence) }\end{array}$ & English & $\begin{array}{l}\text { Spanish, Ma- } \\
\text { lay, Korean, } \\
\text { Persian }\end{array}$ \\
\hline $\begin{array}{l}\text { Performance Assessment of Self-care } \\
\text { Skills (PASS) } \\
\text { Rogers \& Holm (1988) }\end{array}$ & $\begin{array}{l}\text { Adolescence through } \\
\text { old age }\end{array}$ & $\begin{array}{l}\text { This tool is a criterion reference, } \\
\text { client reference, performance-based } \\
\text { and observational }\end{array}$ & & $\begin{array}{l}26 \text { tasks contain: } \\
5 \text { functional mobility } \\
\text { (MOB), } 3 \text { (ADL), } 14 \\
\text { IADL with a cognitive } \\
\text { emphasis (C-IADL), } 4 \\
\text { IADL with a physical } \\
\text { emphasis (P-IADL). }\end{array}$ & 4 point $(0-3)$ ordinal scale. & English & $\begin{array}{l}\text { English } \\
\text { Persian }\end{array}$ \\
\hline
\end{tabular}




\begin{tabular}{|c|c|c|c|c|c|c|c|}
\hline $\begin{array}{l}\text { PROM* } \\
\text { (reference to first article) }\end{array}$ & $\begin{array}{c}\text { Target } \\
\text { population }\end{array}$ & $\begin{array}{l}\text { Mode of administration } \\
\text { (e.g. self-report, interview-based, } \\
\text { parent/proxy Report etc.) }\end{array}$ & $\begin{array}{l}\text { Recall } \\
\text { period }\end{array}$ & $\begin{array}{l}\text { Sub/scales, } \\
\text { Number of items }\end{array}$ & $\begin{array}{c}\text { Range of } \\
\text { scores/scoring }\end{array}$ & $\begin{array}{r}\text { Original } \\
\text { language }\end{array}$ & $\begin{array}{l}\text { Available } \\
\text { translations }\end{array}$ \\
\hline $\begin{array}{l}\text { Texas Functional Living Scale } \\
\text { (TFLS) } \\
\text { Cullum (2001) }\end{array}$ & $\begin{array}{l}\text { Older adults, } \\
\text { especially older adults } \\
\text { with cognitive impair- } \\
\text { ments, } \\
\text { particular Alzheimer's }\end{array}$ & Performance-based & $15-20$ minutes & $\begin{array}{l}24 \text { items in } 4 \text { areas of: } \\
\text { Time, Money, Calcula- } \\
\text { tion, Communication. }\end{array}$ & $\begin{array}{l}\text { Scores range from } 0 \text { to } 5 \text { for each } \\
\text { activity with a maximum possible } \\
\text { score of } 52 \text {. } \\
\text { Total scores and cumulative per- } \\
\text { centages are recorded for each of } \\
\text { the } 4 \text { subscales and an overall T- } \\
\text { score is calculated for the entire } \\
\text { test. Higher scores are suggestive } \\
\text { of better IADL functioning. }\end{array}$ & English & $\begin{array}{l}\text { Only Ameri- } \\
\text { can version of } \\
\text { this tool is } \\
\text { available }\end{array}$ \\
\hline
\end{tabular}

Table 3. Psychometric properties of measures

\begin{tabular}{|c|c|c|c|c|c|c|c|c|c|c|}
\hline \multirow{2}{*}{$\begin{array}{c}\text { PROM } \\
\text { (ref) }\end{array}$} & \multirow{2}{*}{$\begin{array}{l}\text { Country/ } \\
\text { Language }\end{array}$} & \multirow{2}{*}{$\begin{array}{c}\text { Study population/ } \\
\text { No. }\end{array}$} & \multicolumn{4}{|c|}{ Validity } & \multicolumn{4}{|c|}{ Reliability } \\
\hline & & & Face & Content & Construct & Criterion & $\begin{array}{c}\text { Test-retest } \\
\text { [ICC] }\end{array}$ & $\begin{array}{l}\text { Internal consistency } \\
\text { [Cronbach's alpha] }\end{array}$ & $\begin{array}{c}\text { Inter-rater } \\
\text { [Kappa/ICC] }\end{array}$ & Intra-rater \\
\hline \multirow{4}{*}{ 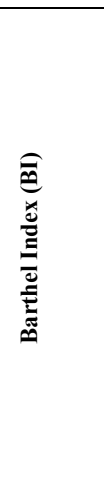 } & $\begin{array}{l}\text { England } \\
\text { (British) } \\
\text { (original version) }\end{array}$ & Stroke/ 258 & + & 0 & $\begin{array}{l}\text { Factor analysis con- } \\
\text { firmed that it is measur- } \\
\text { ing a single domain }(+)\end{array}$ & $?$ & 0 & $\begin{array}{l}\text { Excellent } \\
{[0.90] \quad(+)}\end{array}$ & $\begin{array}{c}\text { Excellent agreement } \\
\text { for total score (in } 15 \\
\text { stroke) } \\
{[\mathrm{k}=0.63-0.85]}\end{array}$ & 0 \\
\hline & $\begin{array}{l}\text { Brazil (Portu- } \\
\text { guese) }\end{array}$ & Stroke/ 57 & + & - & - & 0 & 0 & 0 & $\begin{array}{l}\text { Good agreement for } \\
\text { total score }[\mathrm{k}=0.70]\end{array}$ & 0 \\
\hline & $\begin{array}{l}\text { Netherland } \\
\text { (Dutch) }\end{array}$ & Stroke/ 60 & + & $?$ & $\begin{array}{l}\text { Factor analysis con- } \\
\text { firmed that it is measur- } \\
\text { ing a single domain }(+)\end{array}$ & 0 & $?$ & $\begin{array}{l}\text { Excellent } \\
{[0.96](+)}\end{array}$ & $\begin{array}{l}\text { Excellent agreement } \\
\quad \text { for total score } \\
\quad[\mathrm{K}=0.88](+) \\
\text { Excellent agreement } \\
\text { for individual items } \\
{[\mathrm{K}=0.82] .(+)}\end{array}$ & 0 \\
\hline & $\begin{array}{l}\text { Germany } \\
\text { (German) }\end{array}$ & Stroke/ 72 & + & 0 & 0 & 0 & 0 & 0 & $\begin{array}{l}\text { Excellent agreement } \\
\text { for total score } \\
{[\mathrm{k}=0.93]}\end{array}$ & 0 \\
\hline
\end{tabular}

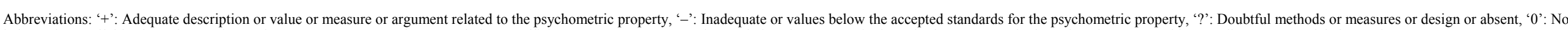
information available about the psychometric property. ICC: Intraclass Correlation Coefficient, K: Kappa, GFI: Goodness of Fit Index, SCI: Spinal Cord Injury, SCL: Spinal Cord Lesion, PD: Parkinson's disease, AD: Alzheimer Disease.

$6 \quad$ http://mjiri.iums.ac.ir

Med J Islam Repub Iran. 2020 (13 Apr); 34:33 


\begin{tabular}{|c|c|c|c|c|c|c|c|c|c|c|}
\hline \multirow{2}{*}{$\begin{array}{c}\text { PROM } \\
\text { (ref) }\end{array}$} & \multirow[t]{2}{*}{ Country/ Language } & \multirow{2}{*}{$\begin{array}{l}\text { Study popu- } \\
\text { lation/No. }\end{array}$} & \multicolumn{4}{|c|}{ Validity } & \multicolumn{4}{|c|}{ Reliability } \\
\hline & & & Face & Content & Construct & Criterion & $\begin{array}{l}\text { Test-retest } \\
\text { [ICC] }\end{array}$ & $\begin{array}{l}\text { Internal consistency } \\
\text { [Cronbach's alpha] }\end{array}$ & $\begin{array}{l}\text { Inter-rater } \\
{[\text { Kappa/ICC }]}\end{array}$ & Intra-rater \\
\hline हैं & USA (American) & $\begin{array}{l}\text { Older adults } \\
\text { in a variety of } \\
\text { care settings }\end{array}$ & 0 & $?$ & 0 & $\begin{array}{l}\text { Convergent or Concurrent } \\
\text { Validity: } \\
\text { High correlation (0.95) } \\
\text { between the Activity index } \\
\text { and the Katz index. }(+)\end{array}$ & 0 & 0 & 0 & 0 \\
\hline 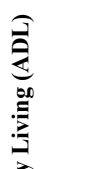 & Brazil (Portuguese) & $\begin{array}{l}\text { Older adults/ } \\
\quad 650\end{array}$ & 0 & 0 & $\begin{array}{l}\text { In factor analysis, only one } \\
\text { factor was extracted (named } \\
\text { independence to ADL) and } \\
\text { other factors were confirmed. } \\
(+)\end{array}$ & 0 & $\begin{array}{c}\text { Excellent } \\
{[0.978]} \\
(+)\end{array}$ & $\begin{array}{c}\text { Good } \\
{[>0.70](+)}\end{array}$ & $?$ & 0 \\
\hline 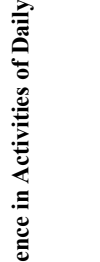 & Turkey (Turkish) & $\begin{array}{l}\text { Older adults/ } \\
\quad 211\end{array}$ & 0 & 0 & $\begin{array}{l}\text { Exploratory Factor Analysis } \\
\text { high factor loading were ob- } \\
\text { tained for items bathing, dress- } \\
\text { ing, toileting and transferring. } \\
\text { Maintaining continence and } \\
\text { feeding correlated less with } \\
\text { other items and the total scale. } \\
(+)\end{array}$ & $\begin{array}{l}\text { Convergent Validity: } \\
\text { Strong associations be- } \\
\text { tween Katz ADL, BI and } \\
\text { SF-36 PF } \quad[r=0.988, \\
\mathrm{p}<0.001 \quad \text { and } \mathrm{r}=0.674, \\
\mathrm{p}<0.001] \quad(+)\end{array}$ & $\begin{array}{c}{[1.000]} \\
(+)\end{array}$ & $\begin{array}{c}\text { Good } \\
{[0.838](+)}\end{array}$ & $\begin{array}{c}\text { Excellent } \\
\text { ICC }=0.999(+)\end{array}$ & 0 \\
\hline 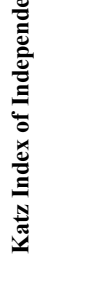 & Iran (Persian) & Stroke/ 87 & 0 & $?$ & $\begin{array}{l}\text { Factor analysis of the Persian } \\
\text { Katz Index indicated two fac- } \\
\text { tors including Motion (bathing, } \\
\text { toileting, and transferring) and } \\
\text { Self-care (dressing, bowel \& } \\
\text { bladder control, and feeding). } \\
\text { (+) }\end{array}$ & $\begin{array}{l}\text { Criterion Validity: } \\
\text { Excellent correlation with } \\
\text { Persian KI and BI. } \\
(\rho=0.92, \quad p<0.001) . \text { Also, } \\
\text { an Excellent }(\rho=0.93) \text { and } \\
\text { High }(\rho=0.88) \text { correlations } \\
\text { between the Persian KI } \\
\text { with self-care and motion } \\
\text { scores of BI. }(+)\end{array}$ & 0 & $\begin{array}{c}\text { Good } \\
{[0.79](+)}\end{array}$ & $\begin{array}{c}{\left[\mathrm{ICC}_{2.1}=0.93\right.} \\
\mathrm{p}<0.001] \\
(+)\end{array}$ & $\begin{array}{c}{\left[\mathrm{ICC}_{2,1}=0.8\right.} \\
3, \mathrm{p}<0.001 \\
(+)\end{array}$ \\
\hline 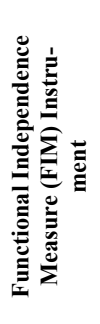 & English & Stroke/? & 0 & + & $\begin{array}{l}\text { Construct validity was assessed } \\
\text { by examining the FIM scores of } \\
11,102 \text { patients upon their en- } \\
\text { tering and leaving a rehabilita- } \\
\text { tion facility. Results showed } \\
\text { that there was significant dis- } \\
\text { crimination between admission } \\
\text { and discharge, in the expected } \\
\text { direction }(\mathrm{p}<0.001)(+)\end{array}$ & $\begin{array}{l}\text { Moderate to High concur- } \\
\text { rent validity of the FIM } \\
\text { with other measures has } \\
\text { been reported, including } \\
\text { correlation with the } \\
\text { Barthel Index (r=0.84), } \\
\text { Katz's Index of ADL } \\
(\mathrm{r}=0.68) \text { and Spitzer's } \\
\text { Quality of Life Index } \\
(\mathrm{r}=0.45)(+)\end{array}$ & 0 & 0 & $\begin{array}{c}\text { High } \\
\text { [ICC: mean of } \\
0.92](+)\end{array}$ & 0 \\
\hline
\end{tabular}


M. Pashmdarfard, et al.

\begin{tabular}{|c|c|c|c|c|c|c|c|c|c|c|}
\hline \multirow{2}{*}{$\begin{array}{c}\text { PROM } \\
\text { (ref) }\end{array}$} & \multirow[t]{2}{*}{ Country/ Language } & \multirow{2}{*}{$\begin{array}{l}\text { Study popula- } \\
\text { tion/No. }\end{array}$} & \multicolumn{4}{|c|}{ Validity } & \multicolumn{4}{|c|}{ Reliability } \\
\hline & & & Face & Content & Construct & Criterion & $\begin{array}{l}\text { Test-retest } \\
\text { [ICC] }\end{array}$ & $\begin{array}{l}\text { Internal consistency } \\
\text { [Cronbach's alpha] }\end{array}$ & $\begin{array}{l}\text { Inter-rater } \\
\text { [Kappa/ICC] }\end{array}$ & Intra-rater \\
\hline \multirow{9}{*}{ 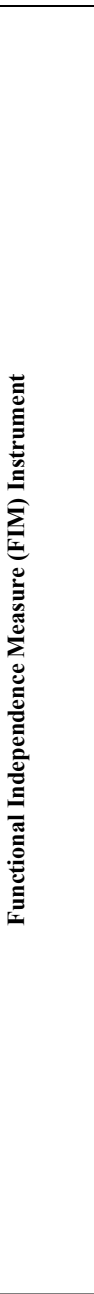 } & Germany (German) & Stroke/ 48 & 0 & + & 0 & $\begin{array}{l}\text { Convergent Validity: } \\
\text { Strong correlation with Katz } \\
\text { ADL, BI }[\mathrm{r}=0.97, \mathrm{p}<0.001 \\
\text { and } \mathrm{r}=0.69, \mathrm{p}<0.001](+)\end{array}$ & 0 & $\begin{array}{l}\text { Excellent } \\
{[0.95](+)}\end{array}$ & 0 & 0 \\
\hline & Italy (Italian) & $\mathrm{SCL} / 103$ & ? & + & 0 & $\begin{array}{l}\text { Validity of i-SCIM } 3 \text { was } \\
\text { confirmed by the close } \\
\text { correlation with FIM results } \\
\text { both at admission and dis- } \\
\text { charge }(\mathrm{r}=0.91, \mathrm{p}<0.01)(+)\end{array}$ & $\begin{array}{l}\text { Excellent } \\
{[0.99](+)}\end{array}$ & $\begin{array}{l}\text { Excellent } \\
{[0.91](+)}\end{array}$ & {$[\mathrm{K}>89 \%]$} & 0 \\
\hline & Spain (Spanish) & $\mathrm{SCl} / 64$ & 0 & + & 0 & $\begin{array}{l}\text { Convergent Validity: } \\
\text { Validity of Spanish version } \\
\text { of FIM was confirmed by } \\
\text { the close correlation with } \\
\text { FIM ( } \mathrm{r}=0.94, \mathrm{p}<0.0001)(+)\end{array}$ & $\begin{array}{c}\text { Excellent } \\
{[>0.97](+)}\end{array}$ & $\begin{array}{l}\text { Excellent } \\
{[0.93](+)}\end{array}$ & {$[\mathrm{K}>0.90]$} & 0 \\
\hline & Sowed (Swedish) & Stroke/ 52 & 0 & - & $?$ & 0 & $\begin{array}{c}\text { Good } \\
{[0.89](+)}\end{array}$ & $\begin{array}{l}\text { Excellent } \\
{[0.91](+)}\end{array}$ & $\begin{array}{l}{\left[\mathrm{ICC}_{2.1}=0.90\right.} \\
\mathrm{p}<0.001](+)\end{array}$ & 0 \\
\hline & Brazil (Portuguese) & Stroke/ 61 & 0 & + & $?$ & 0 & $\begin{array}{c}\text { Good } \\
{[0.89](+)}\end{array}$ & 0 & $0.51<\mathrm{K}<0.90(+)$ & ? \\
\hline & Finland (Finish) & Stroke/ 65 & 0 & 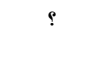 & 0 & $?$ & $?$ & $\begin{array}{l}\text { Excellent } \\
{[0.93](+)}\end{array}$ & $\begin{array}{l}{\left[\mathrm{CC}_{2.1}=0.92\right.} \\
\mathrm{p}<0.001]\end{array}$ & 0 \\
\hline & Africa (African) & Stroke/ 44 & 0 & + & 0 & $\begin{array}{l}\text { Convergent Validity: } \\
\text { Validity of African version } \\
\text { of FIM was confirmed by } \\
\text { the close correlation with } \\
\text { FIM }(\mathrm{r}=0.90, \mathrm{p}<0.0001)\end{array}$ & $\begin{array}{l}\text { Excellent } \\
{[0.98](+)}\end{array}$ & 0 & $\begin{array}{l}{[\mathrm{K}=0.64(0.55-} \\
0.71)](+)\end{array}$ & 0 \\
\hline & Turkey (Turkish) & $\begin{array}{l}\text { consecutive } \\
\text { Stroke/ } 51 \\
\text { SCI/ } 62\end{array}$ & 0 & $?$ & $\begin{array}{l}\text { Construct validity showed ex- } \\
\text { pected associations with the } \\
\text { impairment scales. }(+)\end{array}$ & $\begin{array}{l}\text { Convergent validity: } \\
\text { In the SCI group, total and } \\
\text { subscale scores of motor } \\
\text { FIM were significantly } \\
\text { related to the } \\
\text { ASIA Impairment (Kruskal- } \\
\text { Wallis test, } p<0.05 \text { ). } \\
\text { No correlation was observed } \\
\text { between cognitive FIM and } \\
\text { ASIA Motor/sensory scores } \\
\text { in stroke group. }(+)\end{array}$ & $\begin{array}{c}\text { Excellent } \\
\text { ICC for motor FIM was= } \\
0.90 \text { and for cognitive } \\
\text { FIM was }=0.98 \text { in SCI, } \\
\text { and for motor FIM } \\
\text { was } 0.93 \text { and for cogni- } \\
\text { tive FIM was }=0.92 \text { in } \\
\text { stroke }(+)\end{array}$ & $\begin{array}{c}\text { Excellent } \\
{[0.934} \\
\text { for motor FIM } 0.983 \text { for } \\
\text { cognitive FIM }] \\
(+)\end{array}$ & $\begin{array}{l}\mathrm{k}>0.48 \text { for } \mathrm{SCI} \\
\text { and } \\
\mathrm{K}>0.44 \text { for Stroke }\end{array}$ & 0 \\
\hline & France (French) & $\begin{array}{l}\text { consecutive } \\
\text { Stroke/ } 127\end{array}$ & 0 & 0 & $\begin{array}{l}\text { First factor corresponded to } \\
\text { mobility and locomotion items } \\
\text { (sub-scores } 3 \text { and } 4 \text { ); the second } \\
\text { factor corresponded to cognitive } \\
\text { items (sub scores } 5 \text { and } 6 \text { ); the } \\
\text { third factor corresponded to the } \\
\text { first sub-score (self-care); and the } \\
\text { fourth factor was explained by } \\
\text { the main contribution of sphinc- } \\
\text { ter items (sub score 2). (+) }\end{array}$ & 0 & 0 & $\begin{array}{l}\text { Excellent } \\
{[0.93](+)}\end{array}$ & 0 & 0 \\
\hline
\end{tabular}

$8 \quad$ http://mjiri.iums.ac.ir

Med J Islam Repub Iran. 2020 (13 Apr); 34:33. 
M. Pashmdarfard, et al.

\begin{tabular}{|c|c|c|c|c|c|c|c|c|c|c|}
\hline \multirow{2}{*}{$\begin{array}{c}\text { PROM } \\
\text { (ref) }\end{array}$} & \multirow[t]{2}{*}{ Country/ Language } & \multirow{2}{*}{$\begin{array}{l}\text { Study popula- } \\
\text { tion/No. }\end{array}$} & \multirow[b]{2}{*}{ Face } & \multicolumn{3}{|c|}{ Validity } & \multicolumn{4}{|c|}{ Reliability } \\
\hline & & & & Content & Construct & Criterion & $\begin{array}{l}\text { Test-retest } \\
\text { [ICC] }\end{array}$ & $\begin{array}{l}\text { Internal consisten- } \\
\text { cy } \\
\text { [Cronbach's alpha] }\end{array}$ & $\begin{array}{c}\text { Inter-rater } \\
\text { [Kappa/ICC] }\end{array}$ & Intra-rater \\
\hline 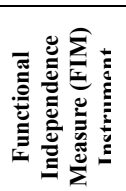 & Iran (Persian) & Stroke/ 40 & $?$ & $?$ & $\begin{array}{l}\text { Construct validity was } \\
\text { supported by a significant } \\
\text { Pearson correlation between } \\
\text { the PFIM and the Persian } \\
\text { Barthel } \quad \text { Index } \\
(\mathrm{r}=0.95 ; \mathrm{p}<0.001)(+)\end{array}$ & 0 & $?$ & $\begin{array}{c}\text { Good to Excellent } \\
{[0.70<} \\
<0.96](+)\end{array}$ & $\begin{array}{l}\text { Excellent } \\
{[\text { ICC }=0.88-0.98]} \\
(+)\end{array}$ & 0 \\
\hline \multirow{2}{*}{ 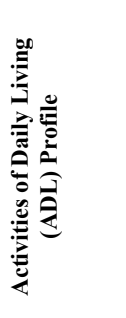 } & Canada (Canadian) & Stroke/? & - & + & $\begin{array}{l}\text { Significant correlations } \\
\text { between } 5 \text { tasks of the ADL } \\
\text { Profile related to personal } \\
\text { care and corresponding } \\
\text { tasks of the Functional } \\
\text { Independence Measure } \\
\text { (Kendall's tau-c }=0.40-0.73 \text {; } \\
\text { p }<0.001) \text {. }(+) \text { ( }\end{array}$ & 0 & 0 & 0 & 0 & 0 \\
\hline & France (French) & Stroke/? & 0 & 0 & 0 & 0 & 0 & 0 & $\begin{array}{l}\text { Adequate } \\
{[\mathrm{k}=0.58-0.68]} \\
(+)\end{array}$ & 0 \\
\hline \multirow{5}{*}{ 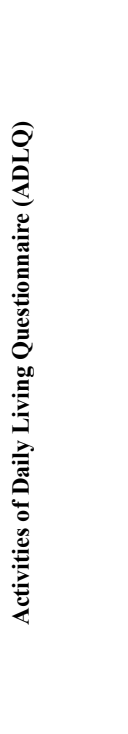 } & USA (American) & Alzheimer/ 140 & 0 & ? & 0 & $\begin{array}{l}\text { Concurrent Validity: } \\
\text { It is high with other measures of temporal } \\
\text { decline in patients with probable Alzheimer } \\
\text { disease and other forms of dementia. } \\
(+)\end{array}$ & $\begin{array}{l}\text { ADLQ has high test- } \\
\text { retest reliability and is } \\
\text { consistent with other } \\
\text { measures of temporal } \\
\text { decline in patients with } \\
\text { Alzheimer disease and } \\
\text { other forms of demen- } \\
\text { tha. [r=0.16; p=0.40] } \\
(+)\end{array}$ & $\begin{array}{c}\text { Good } \\
{[0.86](+)}\end{array}$ & 0 & 0 \\
\hline & China (Chinese) & $\begin{array}{l}\text { Community- } \\
\text { dwelling indi- } \\
\text { viduals with } \\
\text { Dementia/ } 125\end{array}$ & $?$ & $?$ & $\begin{array}{l}\text { Factor analysis yielded } 6 \\
\text { factors }\end{array}$ & $\begin{array}{l}\text { Convergent Validity: } \\
\text { Significant negative correlation between the } \\
\text { total score of the ADLQCV and the CDAD } \\
\text { total score }(r=-0.917, p<0.001) \text {. (+) }\end{array}$ & $\begin{array}{l}\text { Excellent } \\
{[0.998](+)}\end{array}$ & $\begin{array}{c}\text { Good } \\
{[0.81](+)}\end{array}$ & $\begin{array}{c}\text { Excellent } \\
\text { [ICC }=0.997]\end{array}$ & ? \\
\hline & Spain (Spanish) & Dementia/ 40 & 0 & 0 & $\begin{array}{l}\text { Factor analysis was indicat- } \\
\text { ed } 6 \text { factors. }(+)\end{array}$ & $\begin{array}{l}\text { Concurrent Validity: } \\
\text { Significant correlations with } \mathrm{CDR} \text { and } \\
\text { FAQ, both } \mathrm{p}<0.001) .(+)\end{array}$ & 0 & $\begin{array}{c}\text { Good } \\
{[0.88](+)}\end{array}$ & 0 & 0 \\
\hline & Brazil (Brazilian) & Alzheimer/ 60 & 0 & + & $\begin{array}{l}6 \text { factors were extracted as } \\
\text { expected like the original } \\
\text { measurements. }(+)\end{array}$ & $\begin{array}{l}\text { Inversely significant correlation }(-0.793) \text { at } \\
\text { the } 5 \% \text { level between the instruments used } \\
\text { in the data collection, which explains the } \\
62 \% \text { total variance. ( }+ \text { ) }\end{array}$ & 0 & $\begin{array}{c}\text { Good } \\
{[0.759](+)}\end{array}$ & 0 & 0 \\
\hline & Chile (Chilean) & Dementia/ 31 & ? & + & 0 & $\begin{array}{l}\text { Convergent Validity: } \\
\text { ADLQ exhibits significant correlations } \\
(\mathrm{p}<0,0001) \text { with other ratings for functional } \\
\text { capacity, measurements for dementia } \\
\text { severity and global cognitive efficiency } \\
\text { tests. }(+)\end{array}$ & 0 & $\begin{array}{c}\text { Good } \\
{[0.802](+)}\end{array}$ & 0 & 0 \\
\hline
\end{tabular}


M. Pashmdarfard, et al.

\begin{tabular}{|c|c|c|c|c|c|c|c|c|c|c|}
\hline \multirow{2}{*}{$\begin{array}{l}\text { PROM } \\
\text { (ref) }\end{array}$} & \multirow{2}{*}{ Country/ Language } & \multirow{2}{*}{$\begin{array}{l}\text { Study popula- } \\
\text { tion/No. }\end{array}$} & \multirow[b]{2}{*}{ Face } & \multicolumn{3}{|c|}{ Validity } & \multicolumn{4}{|c|}{ Reliability } \\
\hline & & & & Content & Construct & Criterion & $\begin{array}{l}\text { Test-retest } \\
\text { [ICC] }\end{array}$ & $\begin{array}{c}\text { Internal con- } \\
\text { sistency } \\
\text { [Cronbach's } \\
\text { alpha] } \\
\end{array}$ & $\begin{array}{c}\text { Inter-rater } \\
\text { [Kappa/ICC] }\end{array}$ & Intra-rater \\
\hline \multirow{2}{*}{ 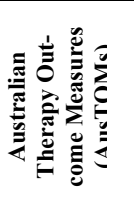 } & Australia (English) & $?$ & + & - & 0 & 0 & $\begin{array}{l}\text { Good to Excellent } \\
{[0.616-0.960](+)}\end{array}$ & 0 & $\begin{array}{l}\text { Moderate to very } \\
\text { High } \\
{[\text { ICC }=0.531-}\end{array}$ & $\begin{array}{c}{[\mathrm{ICC}=0.675-} \\
1.000](+)\end{array}$ \\
\hline & Sowed (Swedish) & $?$ & 0 & 0 & 0 & 0 & $\begin{array}{l}\text { Good to Excellent } \\
{[0.705 \text { to } 0.920](+)}\end{array}$ & 0 & $\begin{array}{l}0.922](+) \\
{[I C C=0.762-} \\
0.904] \\
(+)\end{array}$ & $\underset{(+)}{[\mathrm{ICC} \geq 0.745]}$ \\
\hline 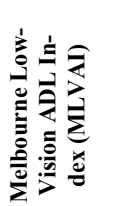 & Australia (English) & $\begin{array}{l}\text { Visual impair- } \\
\text { ment / } 122\end{array}$ & - & + & $?$ & $\begin{array}{l}\text { Convergent Validity: } \\
\text { Moderately to high correlation with vision } \\
\text { impairment }(\mathrm{r}=-0.68, \mathrm{P}<0.001)(+)\end{array}$ & $\begin{array}{c}\text { Good } \\
{[0.88](+)}\end{array}$ & $\begin{array}{l}\text { Excellent } \\
{[0.94](+)}\end{array}$ & $\begin{array}{l}\text { High } \\
{[\text { ICC }=0.95] \quad(+)}\end{array}$ & 0 \\
\hline \multirow[t]{2}{*}{ 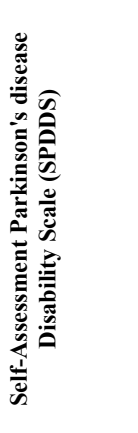 } & England (English) & Parkinson's / 66 & - & + & 0 & - & 0 & 0 & $\begin{array}{l}\text { Complete agree- } \\
\text { ment varied from } \\
41 \% \text { (picking up } \\
\text { an object from the } \\
\text { floor) to } 71 \% \\
\text { (making a cup of } \\
\text { tea, and inserting } \\
\text { and removing an } \\
\text { electrical plug). } \\
\text { The average was } \\
60 \% \text { for the whole } \\
\text { scale (+) }\end{array}$ & 0 \\
\hline & Serbia (Serbian) & $\begin{array}{c}\text { Parkinson's/ } \\
114\end{array}$ & + & + & $\begin{array}{l}\text { According to the ROC } \\
\text { curve, } 70 \% \text { of the total area } \\
\text { was under curve. }(+)\end{array}$ & 0 & $\begin{array}{c}\text { Kendall's concordance } \\
\text { coefficient was }=0.994 \\
(+)\end{array}$ & $\begin{array}{c}\text { Excellent } \\
{[0.984](+)}\end{array}$ & 0 & 0 \\
\hline \multirow{3}{*}{ 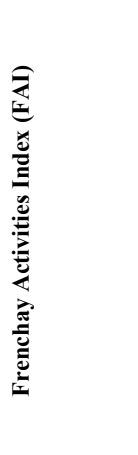 } & England (English) & Stroke/ 60 & - & + & 0 & 0 & - & - & $\begin{array}{l}\text { Kappa statistic } \\
\text { showed } r=0.93 \\
p<0.001)(+)\end{array}$ & 0 \\
\hline & China (Chinese) & Stroke/ 52 & 0 & 0 & 0 & 0 & $?$ & $\begin{array}{l}\text { Kendall tauB } \\
\text { correlation be- } \\
\text { tween the two } \\
\text { sumscores was } \\
0.72(\text { ICC }=0.63 \text { to } \\
0.81)(+)\end{array}$ & 0 & $\begin{array}{l}\text { Weighted } \\
\text { kappa varied } \\
\text { between } 0.36 \\
\text { and } 0.89(+)\end{array}$ \\
\hline & Netherland (Dutch) & $\begin{array}{c}\text { nonstock popu- } \\
\text { lations/ } 602\end{array}$ & 0 & 0 & 0 & $?$ & $\begin{array}{l}\text { Good } \\
\text { (in 57 people) } \\
{[\mathrm{r}=5.96](+)}\end{array}$ & $\begin{array}{l}\text { Excellent } \\
{[0.91](+)}\end{array}$ & $?$ & $\begin{array}{l}91 \% \text { of kappa } \\
\text { coefficients } \\
\text { showed } \\
\text { almost perfect } \\
\text { intra-rater } \\
\text { agreement. } \\
(+)\end{array}$ \\
\hline
\end{tabular}

$10 \quad$ http://mjiri.iums.ac.ir

Med J Islam Repub Iran. 2020 (13 Apr); 34:33. 


\begin{tabular}{|c|c|c|c|c|c|c|c|c|c|c|}
\hline \multirow{2}{*}{$\begin{array}{c}\text { PROM } \\
\text { (ref) }\end{array}$} & \multirow[t]{2}{*}{ Country/ Language } & \multirow{2}{*}{$\begin{array}{l}\text { Study popula- } \\
\text { tion/No. }\end{array}$} & \multirow[b]{2}{*}{ Face } & \multicolumn{3}{|c|}{ Validity } & \multicolumn{4}{|c|}{$\begin{array}{l}\text { Reliability } \\
\end{array}$} \\
\hline & & & & Content & Construct & Criterion & $\begin{array}{l}\text { Test-retest } \\
{[\text { ICC }]}\end{array}$ & $\begin{array}{l}\text { Internal consistency } \\
\text { [Cronbach's alpha] }\end{array}$ & $\begin{array}{c}\text { Inter-rater } \\
{[\text { Kappa/ICC }]}\end{array}$ & Intra-rater \\
\hline 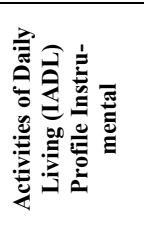 & Canadian & $\begin{array}{l}\text { Moderate or } \\
\text { severe TBI }(16- \\
65 \text { years old }) / \\
30\end{array}$ & - & + & 0 & 0 & 0 & $?$ & $\begin{array}{l}\text { Comparing } \\
\text { ratings of four } \\
\text { raters, } 95 \% \text { of } \\
\text { kappa coeffi- } \\
\text { cients indicated } \\
\text { moderate to } \\
\text { almost perfect } \\
\text { agreement. }(+)\end{array}$ & $\begin{array}{c}94 \% \text { of kappa } \\
\text { coefficients } \\
\text { showed almost } \\
\text { perfect intra-rater } \\
\text { agreement. } \\
(+)\end{array}$ \\
\hline 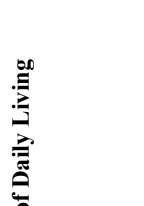 & Spain (Spanish) & $\begin{array}{l}\text { patients aged } 65 \\
\text { or over who } \\
\text { suffered an } \\
\text { accidental fall } \\
\text { with a hip or } \\
\text { wrist fracture as } \\
\text { a result } 1,965\end{array}$ & 0 & ? & $\begin{array}{l}\text { Confirmatory Factor Analy- } \\
\text { sis confirmed the homoge- } \\
\text { neity of the construct validi- } \\
\text { ty }(+)\end{array}$ & $\begin{array}{l}\text { Convergent Validity: } \\
\text { For all correlation coefficients were }>0.40 \\
(+)\end{array}$ & - & $\begin{array}{l}\text { Excellent } \\
{[0.94]}\end{array}$ & 0 & 0 \\
\hline 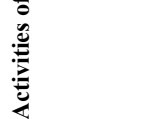 & Malaysia (Malay) & Older adults/ 65 & + & + & 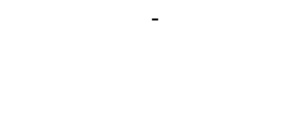 & 0 & $\begin{array}{l}\text { Excellent } \\
{[\mathrm{r}=0.950, \mathrm{p}<0.001]} \\
(+)\end{array}$ & $\begin{array}{l}\text { Good } \\
{[0.838](+)}\end{array}$ & $\begin{array}{l}\text { Excellent } \\
{[\mathrm{r}=0.957} \\
\mathrm{p}<0.001] \\
(+)\end{array}$ & 0 \\
\hline 可 & Korea (Korean), & Older adults/ 65 & + & + & 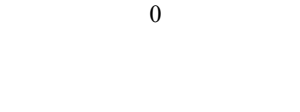 & 0 & $\begin{array}{l}90(\mathrm{p}=0.00)] \\
(+)\end{array}$ & $\begin{array}{l}\text { Excellent } \\
{[>0.90](+)}\end{array}$ & $\begin{array}{l}\text { High agree- } \\
\text { ments for all } 8 \\
\text { items } \\
{[0.75 \sim 1.00](+)}\end{array}$ & - \\
\hline 产 & Iran (Persian) & Dementia/ 60 & + & + & $\begin{array}{l}\text { Construct Validity: } \\
\text { Significant negative rela- } \\
\text { tionship between the partic- } \\
\text { ipants' score in IADL and } \\
\text { FAST }(p<0.001)(+)\end{array}$ & - & $\begin{array}{l}\text { High } \\
{[0.988-0.996]} \\
(+)\end{array}$ & $\begin{array}{l}\text { Between items and } \\
\text { total score } \\
{[0.606>r>0.427] \text { had }} \\
\text { almost an average } \\
\text { power. }(+)\end{array}$ & $\begin{array}{l}\text { High } \\
{[\mathrm{r}=0.961} \\
\mathrm{p}<0.001]\end{array}$ & ? \\
\hline 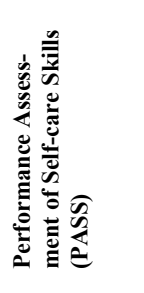 & $\begin{array}{l}\text { USA (English) } \\
\text { Iran (Persian) }\end{array}$ & $\begin{array}{l}\text { Older adults/- } \\
\text { Parkinson's/ } 50\end{array}$ & $\begin{array}{l}+ \\
+\end{array}$ & $\begin{array}{l}+ \\
+\end{array}$ & $\begin{array}{l}- \\
?\end{array}$ & $\begin{array}{l}\quad 0 \\
\text { Rate of agreement: } \\
\text { Significant agreement }(\mathrm{P}=95 \%) \text { between } \\
\text { the scores of } 5 \text { specialists in all } 3 \text { aspects of } \\
\text { independence }(\mathrm{X} 2=5.83, \mathrm{DF}=4) \text {, safety } \\
(\mathrm{X} 2=5.44, \mathrm{DF}=4) \text {, and outcome }(\mathrm{X} 2=2.45 \\
\mathrm{DF}=4)(+)\end{array}$ & $\begin{array}{l}\quad 0 \\
\text { High reliability < } \\
\text { Pearson correlation } \\
\text { coefficient for test- } \\
\text { retest results was } \\
93 \%, 91 \% \text {, and } 91 \% \\
\text { for independence, } \\
\text { safety, and outcome } \\
\text { respectively } \\
(\mathrm{P}=0.01)(+)\end{array}$ & $\begin{array}{l}0 \\
0\end{array}$ & $\begin{array}{l}0 \\
0\end{array}$ & $\begin{array}{l}0 \\
0\end{array}$ \\
\hline 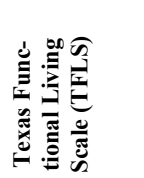 & USA (English) & $\begin{array}{l}\text { Older adults } \\
\text { with cognitive } \\
\text { impairments, in } \\
\text { particular } \\
\text { Alzheimer's/ } 21\end{array}$ & 0 & 0 & 0 & $\begin{array}{l}\text { Convergent Validity: } \\
\text { Strong correlation between TFLE with } \\
\text { Mini-Mental State Examination }(\mathrm{r}=0.92) \\
(+)\end{array}$ & $\begin{array}{l}\text { High } \\
{[\mathrm{r}>0.93, \mathrm{p}<0.001]} \\
(+)\end{array}$ & $\begin{array}{l}\text { Excellent } \\
{[0.92](+)}\end{array}$ & 0 & 0 \\
\hline
\end{tabular}




\section{Activities of Daily Living (ADL) assessment tools}

Barthel Index (BI): Mahoney and Barthel designed this tool in 1955 (15). This tool assesses 10 activities related to Basic ADL consisting: bowels, bladder, grooming, feeding, toilet use, transfer, mobility, dressing, stairs, and bathing. The estimated total score for each person varies from 0 (maximum disability and dependency) to 20 (maximum strength and independence). A total change of at least two points indicates a significant change in the degree of independence and dependence of the individual (16). This tool has been translated into Brazilian, British, Dutch, German, Taiwanese, Turkish, Chinese (Hong Kong) versions (17). Oveisgharan et al. (2006) have validated the Persian version of Barthel Index in 459 stroke patients with a mean age 68.11 years. The correlation between the items was excellent $(\alpha=0.935)$ and the test-retest reliability after two weeks' interval was excellent too (ICC=0.989) (18).

Katz Index of Independence in Activities of Daily Living $(A D L)$ : Katz et al. developed this tool in 1959 (19, 20). This tool is designed to assess the Basic ADL function among older adults in the community and all care settings. The initial version of Katz Index included 8 Basic ADL skills, which adjusted finally to 6 Basic ADL including: bathing, dressing, transfer, toileting, feeding and continence (19). To score this tool, if an older adult is able to perform an activity, he/she gets score 1, and if he/she is unable to do so, will get score 0 . The total score varies between 6 (maximum performance) and 0 (lack of performance). A score 6 indicates the full function, 4 indicates moderate impairment, and 2 or less indicates severe functional impairment (20). This tool has been translated into American, Brazilian, and Turkish versions (21-23). The Persian version of Katz Index has been validated by Azad et al. (2017) in 87 acute stroke patients aged 40-80 years. The internal consistency between the items was good $(\alpha=0.79)$ and the inter-rater reliability was reported excellent $\left(\mathrm{ICC}_{2.1}=0.93, \mathrm{p}<0.001,95 \% \mathrm{CI}\right.$ : 0.89-0.95) (24).

Functional Independence Measure (FIM): It was developed between 1984 and 1987 by the American Academy of Physical Medicine and Rehabilitation and the American Congress of Rehabilitation Medicine and Keith et al. published the final product in 1987. This tool consists of 18 items that evaluate 6 functional areas of the individual. The 13 items of this tool are named as Motor-FIM and its 5 items are named as Cognitive-FIM. Motor-FIM items of this tool is based on the Barthel Index items. This tool is useful for stroke patients in all age groups, and the use of this tool requires training and specialty courses under the supervision of trained people (25). This tool has been translated and validated in German, Italian, Spanish, Swedish, Finnish, Portuguese, African, Turkish, and French versions (26). The Persian version of this instrument has been validated by Naghdi et al. (2016) in 40 stroke patients with an average age of 60 years. The internal consistency of the items was good to excellent $(0.70<\alpha<0.96)$ and the inter-rater reliability has been reported excellent (ICC: 0.88-0.98) (27).

Activities of Daily Living (ADL) Profile: This tool was designed by Dutil et al. in 1990 to evaluate the ADL of traumatic brain injuries. The ADL Profile is a criterion reference instrument consisting of 20 Items. The 17 items of this tool are based on performance-based evaluation (direct observation of the evaluated function), and 3 items are based on semi-structured interviews with the person or other people (individual's caregivers) and completed by a questionnaire. The tool assesses the ADL function in three personal environments (with 6 items in self-care dimensions), Home (with 5 items in the Home dimensions' category), and the Community (with 9 items). The Scoring of this tool varies from 0 (full independency) to 3 (full dependency). It measures individual autonomy in ADL based on four executive performance skills including formulating the goal, planning, carrying out the task, and verifying the initial goal (28). This tool is available in both Canadian and French versions $(29,30)$. The validity and reliability of this tool have not been studied in Iran.

Activities of Daily Living Questionnaire (ADLQ): This tool was developed by Johnson et al. in 2004 to evaluate the ADL performance of individual with cognitive impairment, especially Alzheimer's patients. This tool assesses the ADL performance of individuals with Alzheimer's in six areas of self-care, household care, employment and recreation, shopping and money, travel and communication. Each of the areas of this tool includes a set of activities that individuals or caregivers should answer to them. For scoring each item a 4-point scale from 0 (no problem) to 3 (need help for completion and longterm) is used. Also, there is a score of 9 in each question, which indicates that there is a lack of ability to do activity or not knowing the activity by the individuals. If the person is not familiar with the activity and has not done it in the past, the score of 9 is given to that task. The scoring and calculations of the questionnaire are available in the questionnaire. Individual independence ranges from 0 to $33 \%$ (impairment) to $+67 \%$ (severe impairment) (31). This tool is available in versions of American, Chinese, Spanish, Brazilian and Chilean (31-35). The validity and reliability of this tool have not been studied in Iran.

Australian Therapy Outcome Measures (AusTOMs): Perry et al. (2004) developed the tool for interdisciplinary use between the three rehabilitation teams of Occupational Therapy, Speech Therapy and Physiotherapy. The AusTOMs is a performance-based evaluation tool designed based on the International Classification of Functioning, Disability and Health (ICF) model, and the Occupational Therapy section includes 12 domains. This tool is available in both Australian and Swedish versions (36). The validity and reliability of this tool have not been studied in Iran.

Melbourne Low-Vision ADL Index (MLVAI): Haymes et al. (2001) developed this tool. It evaluates the ADL performance of individuals with visual impairment. This tool is designed as a desk based clinical assessment and includes two areas: the first contains 18 observational items in the field of ADL functions, and the second includes 9 items for self-care activities. Scoring for each item is based on independence, speed, and accuracy of performance on a five descriptive Likert scale (4-0) (37). 
The Persian version of this tool is not available.

Self-Assessment Parkinson's disease Disability Scale (SPDDS): This scale was developed by Brown in 1989 to evaluate the ADL performance of individuals with Parkinson's disease. This scale includes 24 items that assess the ADL performance of individuals with Parkinson's. The scoring scale for this tool is 5 points for each activity and varies from 5 (ability to do work alone and without difficulty) to 1 (unable to do activity) (38). This is available in two British and Serbian versions $(38,39)$. The Persian version of this scale is not available.

\section{Instrumental Activities of Daily Living (IADL) as- sessment tools}

Frenchay Activities Index (FAI): Holbrook and Skilbeck (1983) developed this tool. It evaluates the IADL of stroke patients. The original version of this tool consists of 15 items that cover three areas of domestic chores (items 1 to 5), work / leisure (items 7, 9, 11, 13, 15) and outdoor activities (items 1, 6, 8, 10, 12,14). The score for each item is varied from 1 (lowest level of activity) to 4 (highest level of activity) so that the sum of the minimum points is equal to 15 and the sum of the maximum points is equal to 60 (40). This tool also has two modifications of 13 and 18 items, respectively, by the modified version of 13 items in 2003 by Tooth et al. and the 18-item version in 2004 by Miller et al. $(41,42)$. However, the original version of the questionnaire (15 items) is still used. This tool has been translated and verified in Canadian, Dutch, and Chinese versions (43-45). The Persian version of this tool is not available.

Activities of Daily Living (IADL) Profile Instrumental: Bottar et al. (2010) developed this tool. This tool is actually an upgraded version of the ADL Profile, developed to evaluate the IADL performance of individuals that are closely related to the environmental performance. This tool includes 29 items in 8 areas: putting on outdoor clothes, going to the grocery store, shopping for groceries, preparing a hot meal for guests, having a meal with guests, cleaning up after a meal, getting information and making a budget. This tool is an ecological measure in relation to the degree of individual autonomy in doing activities in the community and at home. The Canadian version of this tool is available and for use requires the presence of workshops held by the developer (46). The Persian version of this tool is not available.

Lawton Instrumental Activities of Daily Living Scale: Lawton and Brody (1969) to assess the independence of older adults in IADL performance developed this scale. This tool includes 8 activities: the ability to use a phone, shopping, meal preparation, housekeeping, laundry, the model of transportation, the responsibility for owns medication, and the ability to handle finance. The scoring scale is zero and one, and the sum of the scores is varied from 0 (low function, dependence) to 8 (high function, independence) (47). Given the items in this tool, women can answer up to 8 questions, but men do not need to answer 3 items related to: meal preparation, housekeeping, and laundry. However, recent studies suggest that it is better for men to respond to these items and questions, as these items together provide a good predictor of the independence and dependence of the older adult on IADL performance (48). This scale has been translated and validated in Australian, Spanish, Malay and Korean versions (4953). This scale was also translated into Persian by Hassani Mehraban et al. (2014); the internal consistency of this questionnaire among the 60 patients with moderate dementia was $(0.427<\mathrm{r}<0.606)$, and the test-retest reliability of this scale after two weeks' interval has been reported excellent (SEM=0.238, r=0.993, CI: 0.988-0.996) (49).

Performance Assessment of Self-care Skills (PASS): This tool is a criterion reference, client reference, performance-based and observational that was developed in 1988 by Rogers and Holm. This tool consists of 26 tasks and 163 sub-tasks. This tool assesses the IADL performance of individuals in four functional areas: functional mobility (including 5 items), Basic ADL (including 3 items), ADL function with an emphasis on physical performance (4 items), ADL function with an emphasis on cognitive function (14 items) (54). This tool has been translated into Persian by Taghizadeh et al. (2008) and its reliability has been evaluated in fifty $45-80$ years old individuals with Parkinson's ( $\mathrm{r}=0.91$, at $\mathrm{P}=0.01)$ (55).

Texas Functional Living Scale (TFLS): Cullum (2001) developed this scale. The TFLS tool assesses the IADL performance of the older adults, especially older adults with cognitive impairments, in particular Alzheimer's. This scale is a performance-based scale that contains 24 items in 4 areas of: time, money, calculation, and communication. Only the American version of this tool is available (56). The Persian version of this tool is not available.

\section{Discussion}

Applying the right and proper assessment tools can help therapists achieve a suitable intervention plan. The purpose of this study is to identify the outcome measures specific to the ADL and IADL for older adults and to investigate the psychometric properties of these measures. Each of the above tools has some advantages and limitations that therapists and researchers must take into account to choose the right and proper assessment tools based on its clinical utility or research purpose.

\section{Activities of Daily Living (ADL) assessment tools}

In the present study, Barthel, Katz, FIM, ADL profile, ADLQ, AusTOMs, MLVAI, and SPDDS tools were introduced as tools for assessing the ADL performance of older adults. Barthel, Katz and FIM tools have good psychometric properties to ADL function of older adults. Researchers report that the Katz questionnaire, given the shortness (number of items) and the general questions specific to the elderly living in the care centers, can be more effective for therapists than the other two scales (Barthel, FIM) in the elderly care centers $(19,20)$. On the other hand, translated versions of the two Barthel and FIM scales are more relevant to the Katz questionnaire, which indicates more use of this tool in assessing the ADL performance of older $(17,26)$. Laura Duffy $(2013)$ in a study showed that the participants reported that Barthel's ques- 
tionnaire was much easier to complete than the FIM questionnaire (17). Another point to consider is that, given the fact that FIM has used the 7-point Likert scale in scoring, it can report the difference between maximum independence and maximum dependence of individuals with more caution and accuracy (25). Among these three tolls the Katz and Barthel are free and don't need any special training to use but FIM requires training and specialty courses under the supervision of trained people to use $(15,20,25)$. The ADL profile and AusTOMs are performance-based tools, although these tools provide much more accurate information than other tools in relation to ADL performance of individuals, but the time duration for completing this assessment takes hours so it is perhaps more difficult for older adults and make them exhausted $(28,36)$, besides the AusTOMs tool is a tool developed for the Australian context (36), and it's better to be used in its context and culture. The ADLQ tool is a comprehensive tool for assessing the ADL performance of individuals with cognitive impairment, in particular Alzheimer's patients, and because it is available in Canadian (31), Chinese (32), Spanish (33), Brazilian (35) and Chilean (35) versions it indicate the high utilization of this tool in assessing the ADL performance of older adults with Alzheimer's. According to that, the psychometric properties of this tool in Iran have not been studied yet. It is suggested that the psychometric properties of this tool be studied in Iran to be used as a useful tool for assessing the ADL performance of older adults by therapists and researchers. Among the ADL assessment tools in older adults the MLVAI tool is specially developed for individuals with visual impairment, and since vision loss is one of the first symptoms in the aging process, this tool can be used to evaluate the ADL performance of older adults with visual impairment. However, given the fact that this tool is also a performance-based tool and requires training, it seems less satisfying than other assessment tools (37). The especial tool for assessing the ADL performance of older adults with Parkinson's disease, is the SPDDS tool, which is specific to the Parkinson's disease and is free and does not require any training (38). The psychometric properties of this scale have not been studied in Iran so it is recommended that the Persian version and the psychometric properties of this tool be studied in Iran.

\section{Instrumental Activities of Daily Living (IADL) assess- ment tools}

In this study, FAI, IADL profile, Lawton, PASS and TFLS tools were reported. The FAI tool is developed specifically for patients with stroke and is usable to examine the progress of the IADL function of patients by 3 to 6 months after the stroke period; so it used in these patients frequently (40). As the Persian version of this tool is not available in Iran, it is suggested that the psychometric properties of this tool in Iran be studied. The IADL Profile questionnaire is an ADL profile modification that can be used for assessing both ADL and IADL performance of older adults. But the limiting factor for using this tool is the need for training courses that requires a fee (46). Among these tools, perhaps the most widely used availa- ble tool in the Persian version is the Lawton questionnaire, which evaluates 8 IADL performance in older adults (47, 48). An implausible point in using this tool in the context of Iran is the existence of a culture of domestic and out-ofhome duties based on gender in Iran. Since some items in the Lawton questionnaire, such as meal preparation and laundry, are the tasks and responsibilities that are most often done by women, the lack of responsiveness to these questions by men leads to a reduction in their rating in the field of IADL performance and this is due to the fact that this reduction is due to cultural differences, not because of the inability to do those tasks. Therefore, it is advisable to be careful about the use of this tool in Iran. The other IADL tool is the PASS tool, which the Persian version of it is also available in Iran $(55,56)$. While the PASS tool is performance-based and has accurate information about the ability of the older adults to do IADL functions, use of this tool is time-consuming and somewhat boring for older adults. Using the TFLS tool, along with the ADLQ tool, both specially developed for Alzheimer's patients, can provide complete information on Alzheimer's performance in ADL and IADL functions $(29,56)$.

\section{Application to Occupational Therapy research and clinical practices}

One of the best ways that occupational therapists can play a role in health management of elderlies is to find a common language between rehabilitation specialists and other disciplines such as physicians. Comprehensive assessment of the needs of elderlies especially the needs related to ADL and IADL using approved assessment tools is important.

This study tried to introduce the ADL and IADL assessment tools of elderlies for the use of occupational therapists and encourage them to use these assessment tools and do there interventions based on the comprehensive evaluation.

\section{Conclusion}

Assessment tools that can report ADL and IADL performance of individuals, especially in older adults, is partly dependent on the culture that dominates the community. Because performance-based tools are tools that give information that is more accurate to therapists and researchers, the use of these tools is better than subjective tools, but most performance-based tools have been developed in other communities and cultures. In addition, they are culture-based tools and need cultural adaptation and also require the cost of training courses for use. Therefore, it is recommended that aging and rehabilitation specialists, especially occupational therapists, try to develop performance-based tools and client-centered tools for assessing the ADL and IADL performance of older adults living in Iran.

\section{Acknowledgement}

Hereby, we appreciate the authorities of the School of Rehabilitation Sciences in Iran University of Medical and the authors announce their gratitude to all those who 
helped us with this research.

\section{Funding}

This research did not receive any grant from funding agencies in the public, commercial, or not-forprofit sectors.

\section{Conflict of Interests}

The authors declare that they have no competing interests.

\section{References}

1. Yavari K, Basakha M, Sadeghi H, Naseri A. Economic Aspects of Ageing. Iran J Ageing. 2015;10(1):92-105.

2. Administration on Aging. A profile of older Americans: 2016. Administration on aging Administration for community living. Retrieved from https://www.giaging.org/documents/.

3. Stuck AE, Beck JC, and Egger M. Preventing disability in elderly people. Lancet. 2004;364:1641-42.

4. Mill'an-Calenti JC, Tub'1o J, Pita-Fern'andez S, Gonz'alez- Abraldes I, Lorenzo T, Fern'andez-Arruty T, et al. Prevalence of functional disability in activities of daily living (ADL), instrumental activities of daily living (IADL) and associated factors, as predictors of morbidity and mortality. Arch Gerontol Geriatr. 2010;50:306-10.

5. American Occupational Therapy Association. Occupational Therapy Practice Framework: Domain and Process 3ed Edition. Am J Occup Ther. 2014;68 (supplement 1):s1-s48.

6. Depp CA, Jeste DV. Definitions and predictors of successful aging: A comprehensive review of larger quantitative studies. Am J Geriatr Psychiatry. 2006;14(1):6-20.

7. Ferrucci L, Guralnik JM, Simonsick E, Salive ME, Corti C, Langlois J. Progressive versus catastrophic disability: A longitudinal view of the disablement process. J Gerontol A-Biol.1996;51A(3):M123-30.

8. Chan RC, Shum D, Toulopoulou T, Chen EY. Assessment of executive functions: Review of instruments and identification of critical issues. Arch Clin Neuropsychol. 2008;23(2):201-16.

9. Hunter EG, Kearney PJ. Occupational therapy interventions to improve performance of instrumental activities of daily living for community-dwelling older adults: A systematic review. Am J Occup Ther. 2018;72:1-9.

10. Albert MS, DeKosky ST, Dickson D, Dubois B, Feldman HH, Fox $\mathrm{NC}$, et al. The diagnosis of mild cognitive impairment due to Alzheimer's disease: Recommendations from the National Institute on Aging-Alzheimer's Association workgroups on diagnostic guidelines for Alzheimer's disease. Alzheimers Dement. 2011;7:270-79.

11. Liu CJ, Chang WP, Chang MC. Occupational therapy interventions to improve activities of daily living for community-dwelling older adults: A systematic review. Am J Occup Ther. 2018;72(4):1-11.

12. Moher D, Liberati A, Tetzlaff J, Altman DG. Preferred reporting Items for systematic reviews and meta-analyses: The PRISMA Statement. PLoS Med. 2009;6(7):e1000097.

13. Mokkink LB, de-Vet HCW, Prinsen CAC, Patrick D, Alonso J, Bouter LM, et al. COSMIN Risk of Bias checklist for systematic reviews of Patient-Reported Outcome Measures. Qual Life Res. 2018;27(5):1171-9.

14. Terwee CB, Jansma EP, Riphagen II, de-Vel HC. Development of a methodological PubMed search filter for finding studies on measurement properties of measurement instruments. Qual Life Res. 2009;18(8):1115-23. doi:10.1007/s11136-009-9528-5

15. Mahoney FI, Barthel DW. Functional evaluation: The Barthel Index. Middle State Medical J. 1965;14:61-65.

16. Taghizadeh G, Martines-Martin P, Meimandi M, Habibi SAH, Jamali S, Dehmiyani A, et al. Barthel Index and modified rank in scale: Psychometric properties during medication phases in idiopathic Parkinson diseases. Ann Phys Rehabil Med. 2019. Doi: 10.1016/j.rehab.2019.08.006.

17. Duffy L, Gajree S, Langhorne PJ. Stott DJ. Quinn T. Reliability (inter-rater agreement) of the Barthel Index for assessment of stroke survivors' systematic review and meta-analysis. Stroke. 2013;44:46268.

18. Oveisgharan S, Shirani S, Ghorbani A, Soltanzade A, Baghaei A, Hosseini S, et al. Barthel Index in a Middle-East Country: Translation, validity and reliability. Cerebrovasc Dis. 2006;22(5-6):350-4.
19. Katz S, Ford AB, Moskowitz RW, Jackson BA, Jaffe MW. Studies of illness in the aged: The Index of ADL: A standardized measure of biological and psychosocial function. $\mathrm{J}$ Am Med Associ. 1963;185(12):914-9.

20. Katz S, Down TD, Cash HR, Grotz RC. Progress in the development of the index of ADL. Gerontologist. 1970;10(1):20-30.

21. Wallace M, Shelkey M. Monitoring functional status in hospitalized older adults. Am J Nurs. 2008;108(4):64-71.

22. Ferretti-Rebustini REL, Balbinotti MAA, Jacob-Filho W, Rebustini F, Suemoto CK, Pasqualucci CAG, et al. Validity of the Katz to assess activities of daily living by informations in neuropathological studies. J School Nursing. 2015;49(6):944-50.

23. Gunes A, Hacer Dogan V, Burcu Balam Y, Erdem K, Ozgur K, Mustafa Kemal K, et al. Validation of Katz Index of independence in activities of daily living in Turkish older adults. Arch Gerontol Geriatr. 2015;61:344-50

24. Azad A, Mohammadinezhad T, Taghizadeh Gh, Lajevardi L. Clinical assessment of activities of daily living in acute stroke: Validation of the Persian version of Katz Index. Med J Islam Repub Iran. 2017;31(1):178-83.

25. Keith RA, Granger CV, Hamilton BB, Sherwin FS. The functional independence measure: a new tool for rehabilitation. Clin Rehab. 1987;1:6-18.

26. Whiting R, Shen, Q, Hung, WT, Cordato D, Chan DKY. Predictors for 5-year survival in a prospective cohort of elderly stroke patients. Acta Neurol Scand J. 2011;124(4):309-16.

27. Naghdi S, Nakhostin-Ansari N, Raji P, Shamili A, Amini M, Hasson S. Cross-cultural validation of the Persian version of the Functional Independence Measure for patients with stroke. Disabil Rehabil. 2016;38(3):289-98.

28. Dutil E1, Forget A, Vanier M, Gaudreault C. Development of the ADL Profile. Occup Ther Health Care. 1990;7(1):7-22.

29. Bottari C, Dassa C, Rainville C, Dutil C. The IADL Profile: Development, content validity, intra- and interrater agreement. Can J Occup Ther. 2010;77(2):90-101.

30. Bottari C, Dutil C, Dassa, C, Rainville C. Choosing the most appropriate environment to evaluate independence in everyday activities: Home or clinic? Australian Occup Ther J. 2006;53:98-106.

31. Johnson N, Barion A, Rademaker A, Rehkemper G, Weintraub S The Activities of Daily Living Questionnaire: A Validation study in patients with Dementia. Alzheimer Dis Assoc Disord. 2004;18(4):223-30.

32. Chu TK, Chung JC. Psychometric evaluation of the Chinese version of the Activities of Daily Living Questionnaire (ADLQ-CV). Int Psychiat Clin. 2008;20(6):1251-61.

33. Muñoz-Neirab C, L-Lópeza O, Riverosb R, Núñez-Huasaf J, Floresb P, Slachevsky A. The Technology-Activities of Daily Living Questionnaire: A version with a technology related subscale. Dement Geriatr Cogn Disord. 2012;33:361-71.

34. Medeiros ME, Guerra RO. Translation, cultural adaptation and psychometric analysis of the Activities of Daily Living Questionnaire (ADLQ) for functional assessment of patients with Alzheimer's disease. Rev Bras Fisioter. 2009;13(3):257-66.

35. Slachevksy A, Muñoz-Neira C, Riveros R. Activities of daily living questionnaire-Chilean version: Psychometric properties and diagnostic utility including a technology sub-scale. Spinal Cord Injury J. 2010;6(4):S492.

36. Perry A. Therapy outcome measures for allied health practitioners in Australia: The AusTOMs. Int J Qua Health Care. 2004;16(4):28591.

37. A. Haymes Sh, W. Johnston A, D. Heyes A. The Development of the Melbourne Low-Vision ADL Index: A measure of vision disability. Invest Ophthalmol Vis Sci. 2001;42(6):1215-25.

38. Brown RG1, MacCarthy B, Jahanshahi M, Marsden CD. Accuracy of self-reported disability in patients with Parkinsonism. Arch Neural Disease. 1989;46(9):955-9.

39. Gazibara T1, Stankovic I, Tomic A, Svetel M, Tepavcevic DK, Kostic VS, et al. Validation and cross-cultural adaptation of the selfassessment disability scale in patients with Parkinson's disease in Serbia. J Neurol. 2013;260(8):1970-7.

40. Holbrook M, Skilbeck CE. An activities index for use with stroke patients. Age Ageing. 1983;12(2):166-70.

41. Tooth LR, McKenna KT, Smith M, O’Rourke P. Further evidence for the agreement between patients with stroke and their proxies on the Frenchay Activities Index. Clin Rehab. 2003;17:656-65. 
42. Miller WC, Deathe AB, Harris J. Measurement properties of the Frenchay Activities Index among individuals with a lower limb amputation. Clin Rehab. 2004;18(4):414-22.

43. J. Schuling R, de Haan M, KH. Groenier L. The Frenchay Activities Index Assessment of Functional Status in stroke patients. Stroke. 1993;24(8):1173-7.

44. Hsueh IP, and Hsieh CL. A revalidation of the Frenchay Activities Index in stroke: A study in Taipei area. J Formos Med Assoc. 1997;6:123-30. [in Chinese].

45. Turnbull JC, Kersten P, Habib M, McLellan L, Mullee MA, George S. Validation of the Frenchay Activities Index in a general population aged 16 years and older. Arch Phys Med Rehabil. 2000;81(8):1034-8.

46. Bottari CL, Dassa C, Rainville CM, and Dutil É. The IADL Profile: Development, content validity, intra- and interrater agreement. Can J Occup Ther. 2010;77(2):90-100.

47. Lawton MP, Brody EM. Assessment of older people: Selfmaintaining and instrumental activities of daily living. Gerontologist. 1969;9(3):179-86.

48. Pearson V. Assessment of function. In R. Kane, \& R. Kane (Eds.). Assessing Older Persons. Measures, Meaning and Practical Applications. 2006:17-48. New York: Oxford University Press.

49. Hassani-Mehraban A, Soltanmohamadi Y, Akbarfahimi M, Taghizadeh Gh. Validity and reliability of the Persian version of Lawton Instrumental Activities of Daily Living Scale in patients with dementia. Med J Islam Repub Iran. 2014;28:25. PMC4153527

50. Itziar-Vergara I, Bilbao A, Orive M, Garcia-Gutierrez S, Navarro GM, Quintana J. Validation of the Spanish version of the Lawton IADL scale for its application in elderly people. Health Qual Life Outcomes. 2012;10:130, 1-7.

51. Kadar M, Ibrahim S, Razaob NA, Siaw Chui-Chai S, Dzalani Harun D. Validity and reliability of a Malay version of the Lawton instrumental activities of daily living scale among the Malay speaking elderly in Malaysia. Australian Occup Ther J. 2018;65:63-8.

52. Young-Kim S, Won-won C, Cho K. The validity and reliability of Korean version of Lawton IADL Index. J Korean Geriatr Soc. 2005;9(1):23-9. (Abstract)

53. Green J, Eagar K, Owen A, Gordon R, Quincy K. Towards a measure of function for home and community care service in Australia: Part 2-evaluation of the screening tool and assessment instrument. Aust J Prim Health. 2006;12(1):81-91.

54. Holm MB, Rogers JC, Hemphill-Pearson B. The performance assessment of self-care skills (PASS). Assessments in Occupational Therapy Mental Health. 2 ed. SLACK; 2008:101-10.

55. Taghizadeh Gh, Shams Aldini A, Karimi H, Rahimzadeh S. Validity and reliability of PASS in evaluating the self-care skills of patients with Parkinson's disease. Salmand: Iranian J Ageing. 2008;3(3,4):4752.

56. Cullum CM, Saine K, Chan LD, Martin-Cook K, Gray KF, Weiner MF. Performance-based instrument to assess functional capacity in dementia: The Texas Functional Living Scale. Neuropsychiatry Neuropsychol Behav Neurol. 2001;14(2):103-8. 